\title{
ON A NEW FUNCTIONAL TRANSFORM IN ANALYSIS: THE MAXIMUM TRANSFORM
}

\author{
BY RICHARD BELLMAN AND WILLIAM KARUSH
}

Communicated by R. P. Boas, June 2, 1961

1. Introduction. In the study of mathematical economics and operations research, we encounter the problem of determining the maximum of the function

$$
F\left(x_{1}, x_{2}, \cdots, x_{N}\right)=f_{1}\left(x_{1}\right)+f_{2}\left(x_{2}\right)+\cdots+f_{N}\left(x_{N}\right)
$$

over the region $R$ defined by $x_{1}+x_{2}+\cdots+x_{N}=x, x_{i} \geqq 0$. Under various assumptions concerning the $f_{i}$, this problem can be studied analytically; cf. Karush $[1 ; 2]$, and it can also be treated analytically by means of the theory of dynamic programming [3].

It is natural in this connection to introduce a "convolution" of two functions $f$ and $g, h=f * g$, defined by

$$
h(x)=\max _{0 \leqq y \leqq x}[f(y)+g(x-y)] .
$$

For purposes of general study, it is more convenient to introduce instead the convolution $h=f \otimes g$ defined by

$$
h(x)=\max _{0 \leqq y \leqq x}[f(y) g(x-y)] .
$$

It is easy to see that the operation $\otimes$ is commutative and associative provided that all functions involved are nonnegative. By analogy with the relation between the Laplace transform and the usual convolution, $\int_{0}^{x} f(y) g(x-y) d y$, it is natural to seek a functional transform

$$
M(f)=F
$$

with the property that

$$
M(f \otimes g)=M(f) M(g),
$$

that is,

$$
H(z)=F(z) G(z)
$$

where $H, F, G$ are the transforms of $h, f, g$ respectively.

We shall show that $M$ exists and has a very simple form. In addition, $M^{-1}$ has a very simple and elegant representation in a number of cases. More detailed discussions and extensions will be presented subsequently. 
2. The maximum transform. Let a transform (1.4) be defined by the equation

$$
F(z)=\max _{x \geqq 0}\left[e^{-x z} f(x)\right], \quad z \geqq 0 .
$$

It will be assumed that $f(x)$ is continuous and nonnegative for $x \geqq 0$. Furthermore, since $F(z)$ is unchanged when $f$ is replaced by its monotone envelope, we shall consider (1) only for monotone nondecreasing $f$.

It is now a straightforward matter to prove (1.5) by the method used in the usual convolution. We have

$$
\begin{aligned}
H(z) & =\max _{x \geqq 0}\left[e^{-x z} \max _{0 \leqq y \leqq x}[f(y) g(x-y)]\right] \\
& =\max _{x \geqq 0} \max _{0 \leqq y \leqq x}\left[e^{-x z} f(y) g(x-y)\right]=\max _{\nu \geqq 0} \max _{x \geqq \nu}[] \\
& =\max _{\nu \geqq 0}\left[f(y) \max _{x \geqq \nu}\left[e^{-x z} g(x-y)\right]\right] \\
& =\max _{y \geqq 0}\left[e^{-y z} f(y) \max _{w \geqq 0}\left[e^{-w z} g(w)\right]\right] \\
& =\max _{y \geqq 0}\left[e^{-y z} f(y)\right] \cdot \max _{w \geqq 0}\left[e^{-w z} g(w)\right]=F(z) G(z)
\end{aligned}
$$

as desired.

To ensure the existence of $F=M(f)$ for $z>0$, it is sufficient to assume that $f$ satisfies a relation of the form $f(x)=O\left[x^{c}\right]$ for $x \geqq 0$ where $c \geqq 0$. The transform $f$ is decreasing and continuous for $z>0$; if $c=0$, this holds for $z \geqq 0$.

3. Inverse operator. The determination of the existence and uniqueness of $M^{-1}$ is of some complexity, and at this time we shall consider only special cases. If for $z>0$, the maximum of $f(x) e^{-x z}$ can be found by differentiation, we have the maximizing value the equation $f^{\prime}(x)-z f(x)=0$. Suppose that this equation possesses a unique solution $x=x(z)$ with $d x / d z \neq 0$ (and hence $<0$ ). For this value of $x$, we have $F(z)=e^{-x z} f(x)$. Differentiating this relation with respect to $x$, we have

$$
F^{\prime}(z) \frac{d z}{d x}=\left(f^{\prime}(x)-z f(x)\right) e^{-x z}-x f(x) e^{-x z} \frac{d z}{d x}=-x f(x) e^{-x z} \frac{d z}{d x} .
$$

Hence,

$$
x=-F^{\prime}(z) / F(z), \quad \text { or } \quad F^{\prime}(z)+x F(z)=0 .
$$


But this is precisely the relation which gives the $z$ minimizing $F(z) e^{x z}$, for fixed $x$. Hence, we have

$$
f(x)=\min _{z \geqq 0} e^{x z} F(z),
$$

the required inversion relation.

A simpler way to obtain this relation is the following. By (2.1), we have, for $x \geqq 0$,

$$
F(z) \geqq e^{-x z} f(x),
$$

whence $F(z) e^{x z} \geqq f(x)$. If there is a one-to-one correspondence between $x$ and $z$ values, we have $\min _{z \geqq 0} F(z) e^{z x} \geqq f(x)$, with equality for one value, whence (3).

\section{Application. Let}

$$
f(x)=\max _{R}\left[f_{1}\left(x_{1}\right) f_{2}\left(x_{2}\right) \cdots f_{N}\left(x_{N}\right)\right],
$$

where $R$ is as in (1.1). Then, inductively,

$$
M(f)=\prod_{i=1}^{N} M\left(f_{i}\right), \quad \text { or } \quad F(z)=\prod_{i=1}^{N} F_{i}(z),
$$

whence formally

$$
f(x)=\min _{z \geqq 0}\left[e^{x z} \prod_{i=1}^{N} F_{i}(z)\right] .
$$

Similarly, if we have a "renewal" equation

$$
f(x)=a(x)+\max _{0 \leqq y \leqq x}[f(y) g(x-y)],
$$

we have a formal solution

$$
f(x)=\min _{z \geqq 0}\left[\frac{e^{x z} A(z)}{1-G(z)}\right],
$$

where $A=M(a), G=M(g)$.

\section{REFERENCES}

1. W. Karush, $A$ queuing model for an inventory problem, Operations Res. vol. 5 (1957) pp. 693-703.

2. - A general algorithm for the optimal distribution of effort, to appear.

3. R. Bellman, Dynamic programming, Princeton, New Jersey, Princeton University Press, 1957.

RAND CORPORATION AND

System Development Corporation 